\title{
ON FIXED POINTS OF COMMUTING FUNCTIONS ${ }^{1}$
}

\section{HASKELL COHEN}

There is a rather well-known conjecture that if $f$ and $g$ are continuous functions on $[0,1]$ to itself which commute (i.e., $f(g(x)$ ) $=g(f(x)))$, then they have a common fixed point. The conjecture is apparently due independently to Eldon Dyer and Allen Shields, and has been generalized by J. R. Isbell [2]. The conjecture is easily verified for polynomials $f$ and $g$ by referring to some work of $\mathrm{J}$. F. Ritt [3] who showed that the only commuting polynomials, aside from some trivial cases are the Tchebycheff polynomials all of which have a common fixed point. This result is stated more explicitly by Block and Thielman [1].

The author has noted that certain functions with broken line graphs, e.g.,

$$
f(x)=\left\{\begin{array}{ll}
2 x & \text { if } 0 \leqq x \leqq \frac{1}{2} \\
2-2 x & \text { if } \frac{1}{2} \leqq x \leqq 1
\end{array}\right\}
$$

and

$$
g(x)=\left\{\begin{array}{ll}
3 x & \text { if } 0 \leqq x \leqq \frac{1}{3} \\
2-3 x & \text { if } \frac{1}{3} \leqq x \leqq \frac{2}{3} \\
3 x-2 & \text { if } \frac{2}{3} \leqq x \leqq 1
\end{array}\right\},
$$

also commute, and that these share with Tchebycheff polynomials (suitably modified by Lemma 1 so that they take $[0,1]$ into $[0,1]$ ) the property he calls fullness. (A function on $[0,1]$ to itself is full if the interval may be subdivided into a finite number of subintervals on each of which the function is a homeomorphism onto $[0,1]$.) In fact every pair of nontrivially commuting continuous functions known to the author are either full, or it is possible to find a subinterval which the restrictions of the functions take onto itself and on which (with the scale properly changed) they are full. The result shown in this note is that if two full functions commute, they have a common fixed point. It is hoped that the result and/or some of the lemmas will be useful in studying the general problem.

Definition. Two functions $f$ and $g$ defined on a set $X$ to itself are said to commute if for each $x \in X$ we have $f(g(x))=g(f(x)) ; f$ and

Received by the editors July 6, 1962 and, in revised form, December 15, 1962.

1 This work was partially supported by the National Science Foundation through grant NSF-G-14085. 
$g$ have a common fixed point if there is an $x \in X$ such that $f(x)=g(x)$ $=x$. We use juxtaposition of functions to indicate composition and may write the commuting property as $f g=g f$.

LEMmA 1. If $f$ and $g$ are functions on the interval $[a, b]$ to itself and $h$ is a homeomorphism of $[a, b]$ onto $[c, d]$, then $h f h^{-1}$ and $h g h^{-1}$ are functions on $[c, d]$ to itself which commute and have a common fixed point if and only if $f$ and $g$ commute and have a common fixed point.

The proof of this lemma is straightforward and is omitted.

LEMMA 2. If there are continuous commuting functions on $[0,1]$ to itself without a common fixed point, then there are also onto functions with these properties.

Proof. Suppose $f$ and $g$ satisfy the hypotheses of the lemma. Let $a_{1}=\max (\inf f$, inf $g)$ and $b_{1}=\min (\sup f, \sup g)$. Since $f$ and $g$ commute, their ranges intersect, and, $a_{1} \leqq b_{1}$. Let $f_{1}$ and $g_{1}$ be $f$ and $g$ restricted to $\left[a_{1}, b_{1}\right]$, respectively; $f_{1}$ and $g_{1}$ take $\left[a_{1}, b_{1}\right]$ into $\left[a_{1}, b_{1}\right]$ for if, for example, $f_{1}(x)>b_{1}$, there is $y \in[0,1]$ such that $g(y)=x$, and $g f(y)=f g(y)=f(x)>b_{1}$ implies $b_{1}<\min (\sup f, \sup g)$. Inductively we let $\quad a_{i}=\max \left(\inf f_{i-1}, \quad \inf g_{i-1}\right), \quad b_{i}=\min \left(\sup f_{i-1}, \quad \sup g_{i-1}\right)$, $f_{i}=f_{i-1} \mid\left[a_{i}, b_{i}\right]$ and $g_{i}=g_{i-1} \mid\left[a_{i}, b_{i}\right]$. The set $\left\{\left[a_{i}, b_{i}\right]\right\}$ forms a nested sequence of closed intervals and has a nonnull intersection. If the intersection were degenerate, $f$ and $g$ would have a common fixed point; hence, the intersection is an interval $[a, b]$, and $\bar{f}=f \mid[a, b]$ and $\bar{g}=g \mid[a, b]$ are onto $[a, b]$. Now letting $h$ be a homeomorphism of $[a, b]$ onto $[0,1]$ and using Lemma 1 , we get $h \bar{f} h^{-1}$ and $h \bar{g} h^{-1}$ as the required functions.

LEMMA 3. If $f$ and $g$ are commuting functions, then $f$ and gf are commuting functions which have a common fixed point if and only if $f$ and $g$ have.

The proof of this lemma is also quite trivial and hence omitted.

Definition. A continuous function $f:[0,1] \rightarrow[0,1]$ will be called full if there exists a partition $P_{f}=\left\{x_{0}, x_{1}, \cdots, x_{n}\right\}$ of $[0,1]$ with $x_{0}=0, x_{i}<x_{i+1}$ and $x_{n}=1$ such that for each $i$ we have $f \mid\left[x_{i}, x_{i+1}\right]$ is a homeomorphism onto $[0,1]$.

It is immediate that the composition of two full functions is full and that $P_{f}$ is unique.

Definition. A partition $P_{f}$ is regular if its subintervals are all the same length. A partition $P_{0}$ refines $P_{f}$ uniformly if each $P_{f}$ interval is the union of the same number of $P_{0}$ intervals. 
LEMMA $4 .^{2}$ If $f_{1}$ and $g_{1}$ are commuting full functions without a common fixed point, there are functions $f$ and $g$ having the same properties and in addition are such that $f(0)=g(1)=0, f(1)=g(0)=1, P_{f}, P_{o}$, and $P_{f o}$ are regular, and $P_{o}$ refines $P_{f}$ uniformly.

Proof. Since $f_{1}(0)=g_{1}(0)=0$ guarantees a common fixed point, we need only (after possibly renaming the functions) consider the cases (1) $f_{1}(0)=0 ; g_{1}(0)=1$ and $(2) f_{1}(0)=1=g_{1}(0)$. In case $(1) f_{1}(1)=f_{1} g_{1}(0)$ $=g_{1} f_{1}(0)=g_{1}(0)=1$; hence $g_{1}(1)$ must be 0 else 1 is a common fixed point. In this case let $f_{2}=f_{1}$ and $g_{2}=g_{1}$. In case (2) $f_{1}(1)=f_{1} g_{1}(0)$ $=g_{1} f_{1}(0)=g_{1}(1)$; therefore to avoid a common fixed point we must have $f_{1}(1)=g_{1}(1)=0$. In this case we let $f_{2}=f_{1} g_{1}$ and $g_{2}=g_{1}$. Now $f_{2}(0)=f_{1} g_{1}(0)=f_{1}(1)=0, g_{2}(0)=g_{1}(0)=1, f_{2}(1)=f_{1} g_{1}(1)=f_{1}(0)=1$ and $g_{2}(1)=g_{1}(1)=0$. In either case let $f_{3}=f_{2}$ and $g_{3}=g_{2} f_{2}$. Clearly $P_{o_{3}}$ refines $P_{f_{3}}$ uniformly, and similarly $P_{f_{3} o_{3}}$ refines $P_{g_{3}}$ uniformly. Now let $\phi$ be any order preserving homeomorphism on $[0,1]$ taking $P_{f_{3} \mathrm{O}_{3}}$ into the corresponding regular partition of $[0,1]$. Let $f=\phi f_{3} \phi^{-1}$ and $g=\phi g_{3} \phi^{-1}$. It is easy to verify that these functions have the required properties.

THEOREM. Commuting full functions must have a common fixed point.

Proof. If not, there exist $f$ and $g$ satisfying Lemma 4. Suppose $P_{f}=\{0,1 / n, 2 / n, \cdots, 1\}$ and $P_{o}=\{0,1 / m, 2 / m, \cdots, 1\} ;$ then $P_{f_{0}}=\{0,1 / m n, 2 / m n, \cdots, 1\}$ and $m$ and $n$ are odd. We adopt the notation that $f_{i}=f \mid[(i-1) / n, i / n]$ and $g_{i}=g \mid[(i-1) / m, i / m]$, let $r=(n+1) / 2, s=(m+1) / 2$, and consider the case when $r$ is odd and $s$ is even (similar arguments can be made for the other cases). Note that $D\left(f_{i} g_{j}\right)$ (the domain of $\left.f_{i} g_{j}\right)$ for each $i$ and $j$ is some subinterval of $P_{f o}$ and, in particular,

$$
\begin{aligned}
& D\left(g_{1} f_{r}\right)=\left[\frac{r-1}{n}, \frac{r-1}{n}+\frac{1}{m n}\right], \\
& D\left(g_{2} f_{r}\right)=\left[\frac{r-1}{n}+\frac{1}{m n}, \frac{r-1}{n}+\frac{2}{m n}\right], \cdots, \\
& D\left(g_{s} f_{r}\right)=\left[\frac{r-1}{n}+\frac{s-1}{m}, \frac{r-1}{n}+\frac{s}{m}\right]=\left[\frac{m n-1}{2 m n}, \frac{m n+1}{2 m n}\right] .
\end{aligned}
$$

2 The author is indebted to the referee for the statement and the shortened proof of this lemma. 
Similarly

$$
\begin{aligned}
& D\left(f_{1} g_{s}\right)=\left[\frac{s-1}{m}, \frac{s-1}{m}+\frac{1}{m n}\right], \\
& D\left(f_{2} g_{s}\right)=\left[\frac{s-1}{m}+\frac{1}{m n}, \frac{s-1}{m}+\frac{2}{m n}\right], \cdots, \\
& D\left(f_{r} g_{s}\right)=\left[\frac{s-1}{m}+\frac{r-1}{n}, \frac{s-1}{m}+\frac{r}{n}\right]=\left[\frac{m n-1}{2 m n}, \frac{m n+1}{2 m n}\right],
\end{aligned}
$$

and we have shown that $D\left(g_{s} f_{r}\right)=D\left(f_{r} g_{s}\right)$. Now $g_{s}$ is continuous and onto $[0,1]$; so its graph must intersect the diagonal of $[0,1] \times[0,1]$ and $g_{s}$ has a fixed point $z_{1}$. Since $D\left(g_{s}\right) \subset D\left(f_{r}\right), z_{1} \in D\left(f_{r}\right)$ and thus $z_{1} \in D\left(f_{r} g_{s}\right)=D\left(g_{s} f_{r}\right)$. Therefore $g_{s} f_{r}\left(z_{1}\right)=f_{r} g_{s}\left(z_{1}\right)=f_{r}\left(z_{1}\right)$ and $z_{2}=f_{r}\left(z_{1}\right)$ is a fixed point of $g_{s}$. Continuing we get a sequence $\left\{z_{p}\right\}$ of fixed points of $g_{s}$ where $z_{p+1}=f_{r}\left(z_{p}\right)$. Since $f_{r}$ is monotone, the sequence $\left\{z_{p}\right\}$ converges to, say, $z$ which clearly is both a fixed point of $f$ and $g$. This contradiction completes the proof.

\section{REFERENCES}

1. H. D. Block and H. P. Thielman, Commutative polynomials, Quart. J. Math. Oxford Ser. (2) 2 (1951), 241-243.

2. J. R. Isbell, Commuting mappings of trees, Bull. Amer. Math. Soc. 63 (1957), 419.

3. J. F. Ritt, Permutable rational functions, Trans. Amer. Math. Soc. 25 (1923), $399-448$.

Louisiana State University 